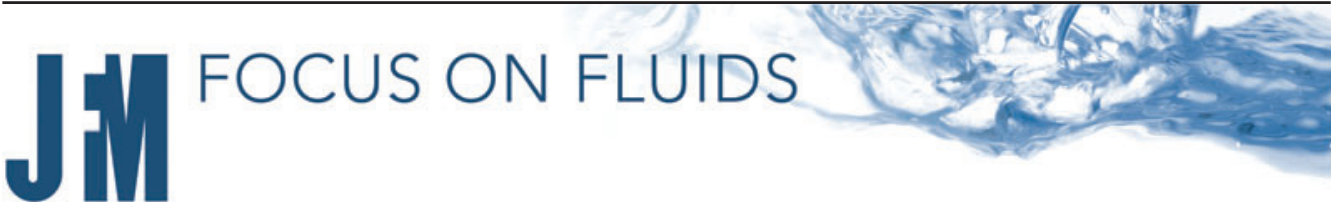

\section{Surfactant transport between foam films}

\author{
Paul Grassia $†$ \\ Department of Chemical and Process Engineering, University of Strathclyde, James Weir Building, \\ 75 Montrose St, Glasgow G1 1XJ, UK
}

Surfactant transport from foam film to foam film is an essential (yet poorly understood) aspect of the viscoplastic yielding behaviour of flowing foam. Recent experimental and modelling work by Bussonnière \& Cantat (J. Fluid Mech., vol. 922, 2021, A25) has, however, helped to advance understanding of the relevant surfactant transport processes: the significance of that work is described herein.

Key words: foams, thin films, lubrication theory, capillary flows

\section{Introduction}

Foams are non-Newtonian fluids exhibiting viscoplastic rheology: they only flow once a yield stress is exceeded. On the bubble scale, the yielding behaviour manifests itself via the so-called $T 1$ topological transformation, which involves bubbles exchanging neighbours (Durand \& Stone 2006). This transformation is described as follows (see figure 1). As a foam is deformed, certain foam films shrink whilst others stretch. Eventually, as a film shrinks away to nothing, bubbles that were originally in contact lose contact with one another. Other bubbles come into contact in their place and a new film is formed. The new film, however, is oriented in such a way as to violate geometrical constraints (the so-called Plateau's laws) on angles at which films in an equilibrium foam must meet. The system must therefore relax to return close to equilibrium. During this relaxation, the newly formed film stretches rapidly and films around it shrink. This then is the fashion in which foam yields.

Thus far the discussion has focussed on the geometric configuration of foam. This, however, ignores an important aspect, namely physical chemistry. Foam films are stabilised by the presence of surfactant molecules on film surfaces. Stretching a foam film that is newly formed after a $T 1$ depletes the surfactant concentration (per unit area) on its surface. Analogously, shrinking neighbouring films causes their surfactant concentration to increase. Surface tension is, however, a function of surfactant concentration: high surfactant concentration implies low surface tension and vice versa. After a T1, Marangoni stresses due to surface tension gradients (Satomi, Grassia \& Oguey 2013) should then drive

\section{$†$ Email address for correspondence: paul.grassia@ strath.ac.uk}




\section{P. Grassia}

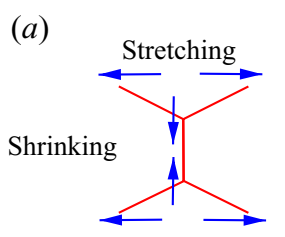

(b)

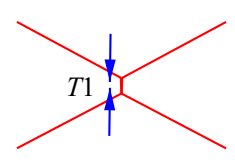

(c)

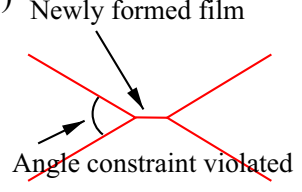

(d)

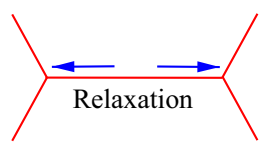

Figure 1. Schematic of a $T 1$ topological transformation.

surfactant from the shrinking (i.e. neighbouring) films to the stretching (i.e. newly formed) one.

Although the physical principle is clear, determining the surfactant flux transported from film to film is challenging. There are hypotheses in the literature (Durand \& Stone 2006; Grassia, Oguey \& Satomi 2012; Satomi et al. 2013) suggesting how a surfactant transport relation might be formulated, but none of them have been subjected to a rigorous fluid mechanical analysis. Indeed, a recent study (Vitasari et al. 2020) modelling surfactant transport within a simple foam structure, considered only surfactant transport along individual films, but simply neglected film-to-film surfactant transport for want of a good model. As described below, recent work by Bussonnière \& Cantat (2021) has the potential to change that.

\section{Experimental study}

Bussonnière \& Cantat (2021) do not solve the long standing question of how surfactant is transported between foam films following a $T 1$ transformation. Instead, given that the $T 1$ is just one mechanism (amongst other possible mechanisms) by which surfactant fluxes on and between foam films can be first generated and then relax, Bussonnière \& Cantat (2021) elect to focus attention on surfactant fluxes more generally. Towards this end, they built an experimental device involving five foam films held in a frame, one central film and four peripheral films, similar to what is sketched in figure $1(d)$. The peripheral films can, however, be stretched or compressed via the action of motors (see also figure 1 within Bussonnière \& Cantat 2021). A configuration is selected in which films on the left start off longer than those on the right, but the former are compressed whilst the latter are stretched. The experiment operates such that the total length of all films is conserved. Effectively what the experiment does then is drive surfactant from left to right, without changing the average surfactant concentration when averaged over all the films.

Using various optical measurements, Bussonnière \& Cantat (2021) determine what happens to film thicknesses, and hence deduce information about stretching or shrinkage of individual film elements. They also observe so called Frankel films extracted from the menisci (usually called Plateau borders in this context) at which three films meet. They can thereby make a distinction between material originally on a given foam film, and material recently added to it that has moved around the Plateau border. Deflection of the Plateau border menisci themselves gives information about surface tensions in the films that meet at the border. Experimental measurements are made both during a driving phase (when motors are switched on) and during a subsequent relaxation phase after motors are switched off.

\section{Modelling study}

The experimental study of Bussonnière \& Cantat (2021) is an impressive achievement in its own right. However, it is also supplemented by an insightful theoretical/ 
modelling analysis. The analysis highlights the fundamental difficulty faced when attempting to model film-to-film surfactant transport, namely 'geometrical frustration'. Because films meet threefold (an odd number) at a Plateau border, there is no way that velocities can be uniform along interfaces and simultaneously uniform across the thickness all films. Shear flow in at least parts of two or more films must therefore occur. For the particular set-up considered (i.e. surfactant driven from left to right with no change to the sum total film length), Bussonnière \& Cantat (2021) argue flow is uniform along and across the central film, so geometrical frustration manifests on those interfaces where peripheral films meet one another.

A clever fluid mechanical asymptotic analysis reveals that far from Plateau borders films are stretched or compressed uniformly via plug-like flows. Very close to Plateau borders there is a static meniscus and also a dynamic meniscus region (both viscous and capillary terms retain relevance in the latter): this situation is familiar in any system involving menisci with fluid motion. The new asymptotic region that Bussonnière \& Cantat (2021) identify, however, is a sheared film region which occurs intermediate between the uniform far field region and the dynamic meniscus region.

A fluid mechanical model based on lubrication theory is presented for how this sheared film region must behave. The essence of the model is that viscous shear stress is matched to Marangoni stress, that surface tension variation is matched to surfactant concentration variation on the surface, and finally that the surfactant flux is conserved, modulo surfactant diffusing from one side of a foam film to another. The model's objective is to derive a relation between the change in the peripheral film tension $\Delta \sigma$ (measured relative to equilibrium film tension, which continues to apply in the central film) and the velocity $U$ at which material is moving at the junction between the central film and a peripheral film: this is then what provides the sought after relation between film-to-film tension difference and surfactant flux.

In addition to $U$, three more characteristic velocity scales are identified, one associated with capillary effects $U_{c}$, one associated with diffusion effects $U_{d}$ and one associated with the Plateau border meniscus $U_{m}$ (treating the Plateau border meniscus as a possible reservoir of surfactant). Bussonnière \& Cantat (2021) go on to derive scaling laws for various limiting cases expressed in terms of relations between the above mentioned velocity scales.

In one extreme limit, it is considered that the Plateau border cannot act as a reservoir at all. If the film under consideration is a peripheral film that happens to be compressed, there cannot be any motion whatsoever at the point where this meets a neighbouring peripheral film. Any surfactant flux carried from the far field towards the Plateau border meniscus must therefore be transferred around the Plateau border meniscus wholly on the interface which connects with the central film. This relies on transferring surfactant diffusively across the film. A linear scaling law between tension difference $\Delta \sigma$ and velocity $U$ is shown to ensue.

Still neglecting any reservoir effect of Plateau border menisci, it is found that the case of a stretched peripheral film sometimes behaves analogously to a compressed one. A linear scaling law between $\Delta \sigma$ and $U$ again results. just with the signs of $\Delta \sigma$ and $U$ being opposite from the compressed case. However, the stretched case also admits a second kind of behaviour. A strongly stretched system with flow being pulled outwards from the point at which two peripheral films meet will have very low surfactant concentration around that point. The requirement to have limited surfactant flux at this particular point is then automatically met, irrespective of the velocity on the film interface. In such a case, the surface tension on one interface of the stretched peripheral film (where it joins the central film) can remain at an equilibrium tension $\sigma_{0}$, but on the opposite interface is $\sigma_{0}+E$, 


\section{P. Grassia}

where $E$ denotes the so called Gibbs elasticity. In the model of Bussonnière \& Cantat (2021) this is the maximum increase in surface tension over and above $\sigma_{0}$, corresponding to a bare surface without surfactant. In this strongly stretched limit, the film tension (the sum of the two surface tensions) then saturates at a value $E$ above the equilibrium film tension, regardless of how large $U$ is, and hence regardless of how much surfactant is being transferred around the Plateau border meniscus from the central film to the stretched peripheral film. This then breaks the symmetry between compression and stretching. Note, however, that any strong stretching here is restricted to just one side of the peripheral film, i.e. the interface which connects to another peripheral film: had stretching occurred on both sides, $\Delta \sigma$ could have been twice as large.

Another interesting limit occurs when the Plateau border meniscus acts as a very effective surfactant reservoir. In the compression case there is no longer any need for surfactant to diffuse from one interface of a film to the other. Compared with the limit discussed earlier, this means that only half of the surfactant flux flowing along the compressed film far from the Plateau border manages to find its way around the Plateau border onto the central film. Diffusive transport within the sheared film turns out to remain an essential feature in the stretched case, however, and the length of the sheared film region grows to accommodate that.

Whether or not Plateau border menisci are able to act as reservoirs affects the ratio between flow velocity on peripheral films and velocity at the junction between the peripheral and central films at the Plateau border itself. The former velocity will be comparable to the latter in the presence of reservoir action, but only half as large in the absence of a reservoir. Bussonnière \& Cantat (2021) also point out that in practice a Plateau border's ability to act as a reservoir is likely to exhaust over time.

\section{Outlook}

Toward the end of their work, Bussonnière \& Cantat (2021) propose a simple illustrative model specifically to describe the behaviour of their five-film device informed by the results they have covered to date. The model describes the stretching of films based on stretching of the Lagrangian material elements that instantaneously make up any given film, plus the film-to-film transfer of material around a Plateau border. Owing to the symmetry of the five-film device (film compression rates opposite and equal to film stretch rates), and assuming tension differences remain linear in velocity around Plateau border menisci, a simple analytical solution is obtained for how tension difference between films (and by implication, surfactant flux around the Plateau border menisci) varies with time. Bussonnière \& Cantat (2021) then suggest a possible route to 'scale up' from their five-film device results to rheology of a three-dimensional foam as a whole.

The work of Bussonnière \& Cantat (2021) does not answer all the open questions about how film-to-film surfactant transport proceeds following a $T 1$ topological transformation nor between foam films more generally. The work relies on a very specific geometry, which is similar to (but not the same as) a $T 1$ geometry. Moreover, the geometry is highly symmetric, so a number of variables can be eliminated on symmetry grounds: the case of disordered foam would not retain those symmetries. Nevertheless insights that Bussonnière \& Cantat (2021) provide will likely prove instrumental in future efforts to gain a better understanding of bubble-scale processes that occur in foams, taking proper account of surfactant physical chemistry. This will in turn advance understanding of foam rheology in general.

Declaration of interests. The author reports no conflict of interest. 


\section{Surfactant transport between foam films}

\section{REFERENCES}

Bussonnière, A. \& CANTAT, I. 2021 Local origin of the visco-elasticity of a millimetric elementary foam. J. Fluid Mech. 922, A25.

DuRAnd, M. \& StONE, H.A. 2006 Relaxation time of the topological $T 1$ process in a two-dimensional foam. Phys. Rev. Lett. 97, 226101.

Grassia, P., Oguey, C. \& SAtomi, R. 2012 Relaxation of the topological T1 process in a two-dimensional foam. Eur. Phys. J. E 35, 64.

Satomi, R., Grassia, P. \& Oguey, C. 2013 Modelling relaxation following T1 transformations of foams incorporating surfactant mass transfer by the Marangoni effect. Colloids Surf. A 438, 77-84.

Vitasari, D., Cox, S., Grassia, P. \& Rosario, R. 2020 Effect of surfactant redistribution on the flow and stability of foam films. Proc. R. Soc. Lond. Ser. A 476, 20190637. 